\title{
Front Matter: Volume 9829
}

, "Front Matter: Volume 9829," Proc. SPIE 9829, Radar Sensor Technology XX, 982901 (23 June 2016); doi: 10.1117/12.2245452

SPIE. Event: SPIE Defense + Security, 2016, Baltimore, MD, United States 


\section{PROCEEDINGS OF SPIE}

\section{Radar Sensor Technology XX}

Kenneth I. Ranney

Armin Doerry

Editors

18-21 April 2016

Baltimore, Maryland, United States

Sponsored and Published by

SPIE 
The papers in this volume were part of the technical conference cited on the cover and title page. Papers were selected and subject to review by the editors and conference program committee. Some conference presentations may not be available for publication. Additional papers and presentation recordings may be available online in the SPIE Digital Library at SPIEDigitallibrary.org.

The papers reflect the work and thoughts of the authors and are published herein as submitted. The publisher is not responsible for the validity of the information or for any outcomes resulting from reliance thereon.

Please use the following format to cite material from these proceedings:

Author(s), "Title of Paper," in Radar Sensor Technology XX, edited by Kenneth I. Ranney, Armin Doerry, Proceedings of SPIE Vol. 9829 (SPIE, Bellingham, WA, 2016) Six-digit Article CID Number.

ISSN: 0277-786X

ISSN: 1996-756X (electronic)

ISBN: 9781510600706

Published by

SPIE

P.O. Box 10, Bellingham, Washington 98227-0010 USA

Telephone +1 3606763290 (Pacific Time) · Fax +1 3606471445

SPIE.org

Copyright (C) 2016, Society of Photo-Optical Instrumentation Engineers.

Copying of material in this book for internal or personal use, or for the internal or personal use of specific clients, beyond the fair use provisions granted by the U.S. Copyright Law is authorized by SPIE subject to payment of copying fees. The Transactional Reporting Service base fee for this volume is $\$ 18.00$ per article (or portion thereof), which should be paid directly to the Copyright Clearance Center (CCC), 222 Rosewood Drive, Danvers, MA 01923. Payment may also be made electronically through CCC Online at copyright.com. Other copying for republication, resale, advertising or promotion, or any form of systematic or multiple reproduction of any material in this book is prohibited except with permission in writing from the publisher. The CCC fee code is 0277-786X/16/\$18.00.

Printed in the United States of America.

Publication of record for individual papers is online in the SPIE Digital Library.

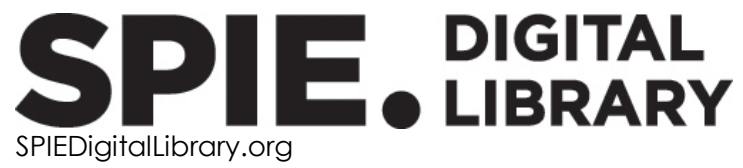

Paper Numbering: Proceedings of SPIE follow an e-First publication model. A unique citation identifier (CID) number is assigned to each article at the time of publication. Utilization of CIDs allows articles to be fully citable as soon as they are published online, and connects the same identifier to all online and print versions of the publication. SPIE uses a six-digit CID article numbering system structured as follows:

- The first four digits correspond to the SPIE volume number

- The last two digits indicate publication order within the volume using a Base 36 numbering

system employing both numerals and letters. These two-number sets start with 00, 01, 02, 03, 04, 05, 06, 07, 08, 09, OA, OB ... 0Z, followed by 10-1Z, 20-2Z, etc. The CID Number appears on each page of the manuscript. 


\title{
Contents
}

\author{
vii Authors \\ ix Conference Committee
}

\section{SESSION $1 \quad$ ALGORITHMS AND PROCESSING I}

982902 Automatic polar ice thickness estimation from SAR imagery [9829-1]

982903 Exploiting synthetic aperture radar imagery for retrieving vibration signatures of concealed machinery [9829-2]

982904 Multistatic passive coherent location using multilateration techniques [9829-4]

982905 Frequency notching effects on GPR imagery while operating in crowded spectrum scenarios [9829-5]

\section{SESSION 2 PROGRAMS AND SYSTEMS}

982906 Context-sensitive design and human interaction principles for usable, useful, and adoptable radars [9829-6]

982907 Second generation of AVTIS FMCW millimeter wave radars for mapping volcanic terrain [9829-7]

$98290894 \mathrm{GHz}$ pulsed coherent radar for high power amplifier evaluation [9829-8]

982909 Eye safe single aperture laser radar scanners for 3D acquisition [9829-9]

9829 OA FlexSAR, a high quality, flexible, cost effective, prototype SAR system [9829-10]

\section{SESSION 3 APPLICATIONS AND EXPLOITATION}

$9829 \mathrm{OB}$ Polarization differences in airborne ground penetrating radar performance for landmine detection [9829-11]

9829 OD Segmenting and extracting terrain surface signatures from fully polarimetric multilook SIR-C data [9829-13]

9829 OE A millimeter-wave reflectometer for whole-body hydration sensing [9829-14]

9829 OF Microwave reconstruction method using a circular antenna array cooperating with an internal transmitter [9829-15] 
9829 OG Fractal characteristics for binary noise radar waveform [9829-16]

$9829 \mathrm{OH} \quad$ High frequency oscillators for chaotic radar [9829-17]

9829 Ol Investigation of correlation characteristics for random array collaborative beamforming using noise signals [9829-18]

$98290 \mathrm{~J}$ Investigation of target and ground clutter reflections on the correlation between transmitted and received noise signals [9829-19]

\section{SESSION $5 \quad$ ALGORITHMS AND PROCESSING II}

9829 OK Efficient sidelobe ASK based dual-function radar-communications [9829-21]

9829 OL Computationally efficient beampattern synthesis for dual-function radar-communications [9829-22]

\section{SESSION 6 NONLINEAR AND COGNITIVE RADAR}

982900 Phase responses of harmonics reflected from radio-frequency electronics [9829-25]

9829 OP Derivation and validation of the nonlinear radar range equation [9829-26]

$98290 \mathrm{Q}$ Waveform design for cognitive radar: target detection in heavy clutter [9829-27]

9829 OR A dynamic spectrum analysis solution for the characterization of the UHF spectrum [9829-28]

\section{SESSION $7 \quad$ INVITED SESSION ON PROFILES IN RESEARCH AND DEVELOPMENT}

9829 OT Pathfinder radar development at Sandia National Laboratories (Invited Paper) [9829-30]

9829 OU Trends in radar: a U.S. Army Research Laboratory perspective (Invited Paper) [9829-31]

9829 OV Three-dimensional radar imaging techniques and systems for near-field applications (Invited Paper) [9829-32]

\section{SESSION 8 INDOOR/URBAN TARGET DETECTION, LOCALIZATION, AND TRACKING}

9829 OY Feature analysis for indoor radar target classification [9829-35]

$98290 Z$ MIMO radar for through-wall target identification in single and two wall scenarios [9829-36]

iv 
982910 Synthetic aperture radar speckle reduction for circle mode SAR images [9829-37]

982911 A novel photonic beam-space receiver for multi-function radar [9829-38]

982912 Matched filter based iterative adaptive approach [9829-39]

982913 An Implementation of real-time phased array radar fundamental functions on DSP-focused, high performance embedded computing platform [9829-40]

982914 Real-time radar signal processing using GPGPU (general-purpose graphic processing unit) [9829-41]

982915 Circuit models for Salisbury screens made from unidirectional carbon fiber composite sandwich structures [9829-42]

\section{SESSION 10 RADAR MICRODOPPLER}

982916 Some applications of the characteristics non-uniform Doppler to radar [9829-43]

982917 Classification of human activity on water through micro-Dopplers using deep convolutional neural networks [9829-44]

982918 Radar fall detectors: a comparison [9829-45]

982919 Radar fall detection using principal component analysis [9829-46]

9829 1B Radar micro-Doppler based human activity classification for indoor and outdoor environments [9829-48]

\section{SESSION 11 QUANTUM RADAR}

9829 1C The generalized ambiguity function: a bridgework between classical and quantum radar [9829-49]

9829 1D Improving quantum sensing efficiency with virtual modes [9829-50]

9829 IE Clutter attenuation using the Doppler effect in standoff electromagnetic quantum sensing [9829-51]

9829 IF A quantum radar detection protocol for fringe visibility enhancement [9829-52]

9829 1G Quantum seismography [9829-53]

9829 1H Analytical formulation of the quantum electromagnetic cross section [9829-54]

9829 1I Quantum computation of the electromagnetic cross section of dielectric targets [9829-55] 
$98291 \mathrm{~J}$ A new two-step algorithm for spotlight synthetic aperture radar imaging [9829-56]

POSTER SESSION

9829 IK Antenna phase center locations in tapered aperture subarrays [9829-57]

9829 IL Representing SAR complex image pixels [9829-58]

9829 IM Comments on airborne ISR radar utilization [9829-59]

9829 iN Index for surface coherence (ISC): a method for calculating change susceptibility [9829-60]

9829 IP Instantaneous stepped-frequency, non-linear radar part 2: experimental confirmation [9829-65]

$98291 Q \quad$ Urban-area extraction from polarimetric SAR image using combination of target decomposition and orientation angle [9829-66]

9829 IR Fully polarimetric data from the ARL RailSAR [9829-67]

9829 is Distributed transmit beamforming on mobile platforms using high-accuracy microwave wireless positioning [9829-68] 


\section{Authors}

Numbers in the index correspond to the last two digits of the six-digit citation identifier (CID) article numbering system used in Proceedings of SPIE. The first four digits reflect the volume number. Base 36 numbering is employed for the last two digits and indicates the order of articles within the volume. Numbers start with 00, 01, 02, 03, 04, 05, 06, 07, 08, 09, OA, OB...0Z, followed by 10-1Z, 20-2Z, etc.

Adler, Eric D., OU Ahmad, Fauzia, OK, 18, 19

Alexander, David B., Ol

Allebach, Joshua M., OJ

Amin, Moeness G., OK, OL, 18, 19

Atwood, Thomas, 03

Balasingham, llangko, $\mathrm{OF}$

Beal, A. N., OH

Bickel, D. L., 1K

Blake, William, 12

Blakely, J. N., $\mathrm{OH}$

Boashash, Boualem, 18, 19

Brandsema, Matthew J., $1 \mathrm{H}$

Breaux, Nancy A., 16

Broderick, Sean P., $0 Z$

Brown, E. R., OE

Bufler, Travis D., OY

Cai, Jingxiao, 14

Campbell, Justin B., 03

Capron, Barbara, $1 \mathrm{~F}$

Cassidy, Scott L., 07

Castillo, Steven, OT

Christianson, Andrew J., 04

Comberiate, Thomas M., is

Corron, N. J., $\mathrm{OH}$

Dean, R. N., $\mathrm{OH}$

Deroba, Joseph C., 11

Dietlein, Charles, OU

Doerry, Armin, 03, 1K, 1L, 1M

Dogaru, Traian, OB, OU

Dunkel, Ralf, 03

Erol, Baris, 18

Finegan, T. M., 09

Fox, Geoffrey C., 02

Gallacher, Thomas F., 08

Gallagher, Kyle A., OO, OP, OR, OU, IP

Geaga, Jorge V., OD

Gebhardt, Evan T., $\mathrm{OZ}$

Gerstle, Walter H., 03

Govoni, Mark A., OU

Gray, John E., 16, 1C

Hall, Thomas E., OV

Hansen, Jeremiah J., 16

Haslem, Brent, OA

Hassanien, Aboulnasr, OK, OL

Hayat, Majeed M., 03

Hedden, Abigail, OU

Himed, Braham, OI, OJ

Hodkin, Jason E., is
Hu, Mengqi, $1 \mathrm{~J}$

Hunter, Robert I., 08

Jaramillo, Monica, 03

Jensen, Mark, OA

Jitrik, Oliverio, 1D, 1E, 1G, 11

Jokanovic, Branka, 19

Jones, A. Mark, OV

Kaiser, Sean A., 04

Kim, Youngwook, 17

Kirk, Benjamin H., OQ

Kirose, Getachew, IR

Klein, Laura M., 06

Knight, Chad, OA

Koltenbah, Benjamin, $1 \mathrm{~F}$

Kong, Fanxing, 14

Lanzagorta, Marco, 1D, 1E, 1G, 1H, 11

Le, Calvin, $O B$

Le, Truc, ID

Lenzing, Erik H., 15

Li, Bing C., OG

Li, Zhengzheng, 12

Lu, Da, $1 Q$

Macfarlane, David G., 07

Martone, Anthony F., 0O, OP, OQ, OR, OU, IP

Mazzaro, Gregory J., 00, OP, IP

McCormick, K., 09

McGowan, Sean F., 00

McMakin, Douglas L., OV

McNamara, Laura A., 06

Mitchell, Gregory, OU

Moon, Taesup, 17

Musgrove, Cameron, 10

Nanzer, Jeffrey A., is

Narayanan, Ram M., 04, 05, OF, OI, OJ, 0O, OP, OQ,

OR, OY, OZ, 15, 1B, 1H, IP

Nepal, Ramesh, 12

Nolan, P., 09

Palmer, Robert D., 14

Parazzoli, Claudio, IF

Parks, Allen D., 1C

Patel, Ankit, 13

Pérez, Francisco, 03

Phelan, Brian R., 05, 1R

Pooler, Richard K., OR

Prather, Dennis, 11

Qiao, Zhijun G., 1J, 1Q

Rahnemoonfar, Maryam, 02

Ranney, Kenneth I., 05, OU, 1P, IR

Ressler, Marc A., 05 
Riley, Ellioł J., 15

Robertson, Duncan A., 07, 08

Santhanam, Balu, 03

Schuetz, Chris, 11

Sheen, David M., OV

Sherbondy, Kelly D., 05, 00, OP, OQ, OR, 1P, IR

Shi, Shouyuan, 11

Smith, Gregory D., 05

Starodubov, D., 09

Sun, Ligang, $1 \mathrm{~J}$

Tedeschi, Jonathan R., OV

Tran, Jonathan, $1 \mathrm{~N}$

Uhlmann, Jeffrey, 1D, 1E, 1G, 11

Venegas-Andraca, Salvador E., 1D, 1E, 1G, 11

Volfson, L., 09

Wang, Guanyong, $1 \mathrm{~J}$

Weber, Mark, 13

Wiesman, Seth, 11

Wu, Zhilu, 1Q

Yari, Masoud, 02

Yu, Xining, 13

Zaghloul, Amir, OU

Zahrai, Allen, 13

Zenaldin, Matthew, 1B

Zhang, Lei, $1 \mathrm{~J}$

Zhang, W.-D., OE

Zhang, Yan Rockee, 12, 13, 14

Zhang, Yimin D., OK, OL

Zhou, Huiyuan, OF

Zilevu, Kojo S., 1 S

Zou, Bin, 1Q 


\section{Conference Committee}

Symposium Chair

David A. Logan, BAE Systems (United States)

Symposium Co-chair

Donald A. Reago Jr., U.S. Army Night Vision \& Electronic Sensors

Directorate (United States)

Conference Chairs

Kenneth I. Ranney, U.S. Army Research Laboratory (United States)

Armin Doerry, Sandia National Laboratories (United States)

Conference Program Committee

Fauzia Ahmad, Villanova University (United States)

Moeness G. Amin, Villanova University (United States)

Joseph C. Deroba, U.S. Army CERDEC Intelligence and Information

Warfare Directorate (United States)

Mark Govoni, U.S. Army CERDEC Intelligence and Information

Warfare Directorate (United States)

John E. Gray, Naval Surface Warfare Center Dahlgren Division

(United States)

Majeed Hayat, The University of New Mexico (United States)

Chandra Kambhamettu, University of Delaware (United States)

Seong-Hwoon Kim, Raytheon Space \& Airborne Systems

(United States)

James L. Kurtz, University of Florida (United States)

Marco O. Lanzagorta, U.S. Naval Research Laboratory (United States)

Changzhi Li, Texas Tech University (United States)

Jenshan Lin, University of Florida (United States)

Hao Ling, The University of Texas at Austin (United States)

David G. Long, Brigham Young University (United States)

Jia-Jih Lu, General Atomics Aeronautical Systems, Inc. (United States)

Neeraj Magotra, Western New England University (United States)

Anthony F. Martone, U.S. Army Research Laboratory (United States)

Gregory J. Mazzaro, The Citadel (United States)

George J. Moussally, Mirage Systems (United States)

Ram M. Narayanan, The Pennsylvania State University (United States)

Lam H. Nguyen, U.S. Army Research Laboratory (United States)

Hector A. Ochoa, The University of Texas at Tyler (United States)

Zhijun G. Qiao, The University of Texas-Pan American (United States) 
Ann Marie Raynal, Sandia National Laboratories (United States) Jerry Silvious, U.S. Army Research Laboratory (United States)

Brian Smith, U.S. Army Armament Research, Development and Engineering Center (United States)

Helmut Suess, Deutsches Zentrum für Luft- und Raumfahrt e.V. (Germany)

David Tahmoush, U.S. Army Research Laboratory (United States)

Russell Vela, Air Force Research Laboratory (United States)

Berenice Verdin, The University of Texas at El Paso (United States)

Frank Yakos, SELEX Galileo, Inc. (United States)

Yan Rockee Zhang, The University of Oklahoma (United States)

\section{Session Chairs}

Opening Remarks

Armin W. Doerry, Sandia National Laboratories (United States)

Kenneth I. Ranney, U.S. Army Research Laboratory (United States)

1 Algorithms and Processing I

Jerry L. Silvious, U.S. Army Research Laboratory (United States)

2 Programs and Systems

Gregory J. Mazzaro, The Citadel (United States)

3 Applications and Exploitation

Joseph C. Deroba, U.S. Army CERDEC Intelligence and Information Warfare Directorate (United States)

4 Noise and LPI Radar

Yan Rockee Zhang, The University of Oklahoma (United States)

5 Algorithms and Processing II

Lam H. Nguyen, U.S. Army Research Laboratory (United States)

6 Nonlinear and Cognitive Radar

Anthony F. Martone, U.S. Army Research Laboratory (United States)

7 Invited Session on Profiles in Research and Development

Armin W. Doerry, Sandia National Laboratories (United States)

Kenneth I. Ranney, U.S. Army Research Laboratory (United States)

8 Indoor/Urban Target Detection, Localization, and Tracking

Ram M. Narayanan, The Pennsylvania State University (United States)

9 Algorithms and Processing III

Ann Marie Raynal, Sandia National Laboratories (United States) 
10 Radar MicroDoppler

Ram M. Narayanan, The Pennsylvania State University (United States)

11 Quantum Radar

Marco O. Lanzagorta, U.S. Naval Research Laboratory (United States)

Salvador Elias Venegas-Andraca, Tecnológico de Monterrey (Mexico)

12 CS for Radar: Joint Session with Conferences 9829 and 9857

Ram M. Narayanan, The Pennsylvania State University (United States) 
Proc. of SPIE Vol. $9829982901-12$

Downloaded From: https://www.spiedigitallibrary.org/conference-proceedings-of-spie on 25 Apr 2023 Terms of Use: https://www.spiedigitallibrary.org/terms-of-use 